\title{
Dispersal dynamics: Distribution of lady beetles (Coleoptera: Coccinellidae)
}

\author{
VLASTIMIL KŘIVAN \\ Department of Theoretical Ecology, Institute of Entomology, Biology Centre, Academy of Sciences of the Czech Republic, \\ Branišovská 31, 37005 České Budějovice, Czech Republic; e-mail: krivan@entu.cas.cz
}

Key words. Balanced dispersal, Coccinella septempunctata, habitat choice, ideal free distribution, matching principle

\begin{abstract}
This article studies coccinellid dispersal in heterogeneous environments using some hypotheses on dispersal rates that correspond to empirical observations. It is assumed that emigration rates increase with decreasing patch payoff that is measured either as the number of aphids per a lady beetle, or as the number of aphids only. Three scenarios for immigration are considered: individuals choose patches unconditionally, immigration is proportional to patch quality, and immigration is proportional to patch payoff. Coccinellid spatial distributions corresponding to these assumptions are given by a power law. Using some data from the literature on distribution of Coccinella septempunctata it is shown that the model with emigration proportional to the ratio of the number of conspecifics to aphid density and unconditional immigration rates provides the best fit when compared with the other models. This model predicts undermatching where better patches get lower consumer density when compared with the ideal free distribution.
\end{abstract}

\section{INTRODUCTION}

Distribution of consumers with respect to their resources is important for understanding mechanisms regulating species coexistence. Several authors observed (e.g., Turchin \& Kareiva, 1989; Hodek \& Honěk, 1996; Elliott \& Kieckhefer, 2000; Osawa, 2000; Evans \& Toler, 2007) that density of adult lady beetles is positively correlated with aphid density. The question arises about what are the mechanisms that create such correlated distributions. To answer this question the two components that describe animal dispersal, immigration and emigration, must be known.

There is empirical evidence that the time coccinellids remain in experimental plots is positively correlated with patch quality. Banks (1957) and Osawa (2000) observed that lady beetles spend more of their time searching where prey are dense than where prey are sparse. Ives et al. (1993) observed positive correlation between the time females of lady beetle stayed on a fireweed (Epilobium angustifolium L.) stem and aphid density. These authors also observed a similar relationship on a larger spatial scale where scattered individuals of fireweed were manipulated experimentally to harbor varying numbers of aphids. Van der Werf et al. (2000) observed increased residence time and 10-20 times higher abundance of lady beetles in sugar sprayed plots when compared with control plots. As the emigration rate is inversely proportional to the staying time, these works (reviewed in Evans, 2003) provide solid evidence that emigration rate decreases with increasing number of prey. Cardinale et al. (2006) observed a linear negative relationship between the emigration rate of lady beetles and the logarithm of aphid density. However, even when aphids are abundant at a given habitat a significant proportion of coccinellid adults disperses every day (Ives, 1981; Frazer, 1988). Interference between conspecifics can be one of the rea- sons for dispersal. For example, Růžička \& Zemek (2007) observed that coccinellid larvae avoid staying on substrates with tracks of other larvae. These observations suggest that emigration rate may depend on both aphid and conspecific density.

With respect to immigration the situation is less clear. Honěk (1980) (see also Hodek \& Honěk, 1996) estimated that the minimum aphid abundance capable of retaining predators is about 10 aphids per square meter of the crop area. Tracking individual lady beetles Osawa (2000) showed that short range movement of a lady beetle within plants of the same species or genus led to a better habitat only in approx. $44 \%$ of observed cases. However, movement between different sub-populations (i.e., plants of different genus) led to a better habitat in $75 \%$ of observations. Cardinale et al. (2006) observed no correlation between immigration rates and aphid density.

An idealized model describing spatial distribution of consumers in a patchy environment is the Ideal Free Distribution (IFD; Fretwell \& Lucas, 1970). At the IFD, consumer payoff in all occupied patches is the same and the number of immigrants to a given patch must equal the number of emigrants from the patch. Such a pattern of equalized immigration and emigration is called "balanced dispersal" (McPeek \& Holt, 1992; Doncaster et al., 1997; Holt \& Barfield, 2001). As under the IFD number of consumers does not equalize across patches, it is clear that to keep such distribution the probability that an individual from a patch with many conspecifics will disperse must be smaller than is the same probability for an individual in a less densely populated patch. In fact, under balanced dispersal, there is an inverse relation between local population size and per capita dispersal rate so that individuals at good sites are less likely to disperse than those from poor sites (Holt \& Barfield, 2001). Unbalanced dispersal caused by e.g., random animal movements between 
patches leads to a higher consumer density in poor patches and a lower density in good patches when compared with the IFD. This phenomenon is known as "undermatching" (Milinski, 1988; Kennedy \& Gray, 1993; Houston et al., 1995; Jackson et al., 2004). It was shown that unconditional (i.e., random) dispersal should be selected against in spatially heterogeneous environments (Hastings, 1983; Holt, 1985; McPeek \& Holt, 1992).

The Fretwell and Lucas concept is static in the sense that it does not address the question how a population distribution achieves the IFD. Cressman \& Krrivan (2006) studied various dispersal dynamics that lead to the IFD. They proved that if individuals never move to patches with a lower payoff and they do not ignore the best patch then the resulting distribution will correspond to the IFD. In this article I will study dispersal rules that are qualitatively consistent with empirical observations on coccinellids. Using some empirical data on lady beetles distribution I will test which of these dispersal rules leads to a distribution that fits the data best.

\section{MODELS}

In this section I construct models that describe predator dispersal in a heterogeneous environment consisting of $H$ patches. Following Cressman \& Krrivan (2006), consumer distribution dynamics are described by

$\frac{d m_{i}}{d t}=\sum_{j=1}^{H}\left(I_{i j}(m) m_{j}-I_{j i}(m) m_{i}\right)$ for $i=1, \ldots, H$.

Here $I_{i j}$ is the transition probability that an individual currently in patch $j$ disperses to patch $i$ in a unit time interval. By choosing a small enough unit time interval, I will assume that all entries in the dispersal matrix are non-negative and sufficiently small so that $1-\sum_{j=1, j \neq i}^{H} I_{j i}>0$. Because the terms $I_{i i}(m) m_{i}$ describing movement from patch $i$ to itself cancel in model (1), diagonal terms of the dispersal matrix $I$ can be chosen in such a way that the sum of the entries in each column of the migration matrix equals 1 (by setting $\left.I_{i i}(m)=1-\sum_{j=1, j \neq i}^{H} I_{j i}\right)$. Moreover, I will assume that $I_{i j}$ is the product of immigration rate $a_{i}\left(m_{i}\right)$ to patch $i$ and emigration rate $b_{j}\left(m_{j}\right)$ from patch $j$. This means that emigration from a patch and immigration to a patch are assumed to be independent processes. These assumptions lead to the following form of migration dynamics

$\frac{d m_{i}}{d t}=a_{i}\left(m_{i}\right) \sum_{j=1}^{H} b_{j}\left(m_{j}\right) m_{j}-b_{i}\left(m_{i}\right) m_{i} \sum_{j=1}^{H} a_{j}\left(m_{j}\right)$ for $i=1, \ldots, H .(2)$

The first term on the right hand side describes immigration to patch $i$ and the second term emigration from patch $i$. The equilibrium distribution $m^{*}=\left(m_{1}^{*}, \ldots, m_{H}^{*}\right)$ of model (2) satisfies the following equation

$$
\frac{a_{i}\left(m_{i}^{*}\right)}{b_{i}\left(m_{i}^{*}\right) m_{i}^{*}}=C \text { for } i=1, \ldots, H
$$

where constant $C$ is given by

$$
C=\frac{\sum_{j=1}^{H} a_{j}\left(m_{j}^{*}\right)}{\sum_{j=1}^{H} b_{j}\left(m_{j}^{*}\right) m_{j}^{*}} .
$$

Cressman \& Krrivan (2006) proved that provided dispersal matrix changes continuously with changing distribution and that immigration to a patch does not decrease as a payoff in that patch increases and emigration from a patch does not increase as the payoff in that patch increases then the equilibrium distribu- tion is locally asymptotically stable. These assumptions hold for all dispersal models I will consider below.

\subsection{Patch payoff}

I will assume that each patch is characterized by its payoff $V_{i}$ and I will consider two patch payoffs. The first payoff is given by patch quality (measured as aphid abundance $K_{i}$ in the patch) per an individual lady beetle

$$
V_{i}=\frac{K_{i}}{m_{i}} .
$$

Because under the IFD payoffs in all occupied patches must be the same (Fretwell \& Lucas, 1970), payoff (4) leads to the so called "matching principle"

$$
\frac{m_{i}}{m_{j}}=\frac{K i}{K_{j}} \text { for } i, j=1, \ldots, H
$$

(e.g., Parker, 1978, Parker \& Stuart, 1976, Milinski \& Parker, 1991, Sutherland, 1996). Thus, input matching principle is a particular form of the IFD for patch payoffs given by (4). I remark that under the input matching principle all patches will be occupied by lady beetles because as the consumer numbers in a patch decrease patch payoff tends to infinity and individuals start to move there.

As an alternative, I will also consider the case where patch payoff does not depend on the number of conspecifics, i.e.,

$$
V_{i}=K_{i}
$$

In this case, under the IFD only the patch(es) with the highest $K_{i}$ will be occupied. This shows why payoff (4) is preferred to (5) in the literature on the IFD.

\subsection{Emigration}

Ives et al. (1993) and Osawa (2000) observed that the patch staying time of a lady beetle increases with aphid density. Here, I will generalize this observation by assuming that the staying time increases linearly with patch payoff (the proportionality constant cancels out in subsequent calculations and I set it arbitrarily equal to 1$)$. As the emigration rate from the $i$-th patch is inversely proportional to patch payoff (i.e., $b_{i}=\frac{1}{V_{i}}$ ) I get

$$
b_{i}=\frac{m_{i}}{K_{i}}
$$

when $V_{i}$ is given by equation (4) and

$$
b_{i}=\frac{1}{K_{i}}
$$

when $V_{i}$ is given by (5). In both cases, emigration rates from patch $i$ always decrease with increasing number of aphids in that patch.

\subsection{Immigration is unconditional}

Here I assume that the probability of immigrating to any of the $H$ patches is the same and equal to $a_{i}=1 / H$ (assuming that animals can return to the patch they departed from). From (3) it follows that for emigration rates given by (6) the equilibrium distribution satisfies

$$
m_{i}=c \sqrt{K_{i}} \text { for every } i=1, \ldots, H
$$

where $c$ is a constant. Thus, $m_{i} / m_{j}=\sqrt{K_{i}} / \sqrt{K_{j}}$ which is not the IFD. Unconditional immigration leads to "undermatching" where better patches get lower animal numbers when compared with the IFD.

For emigration rates (7) that depend on the aphid density only the equilibrium distribution is

$$
m_{i}=c K_{i} \text { for every } i=1, \ldots, H .
$$

It is interesting to note that despite the fact that immigration is random and emigration rates depend on the patch quality only, the resulting lady beetle distribution corresponds to the Parker's matching principle, thus to the IFD. 
TABLE 1. Best fit parameter(s) for models (8), (9), (11), and (10) using data on coccinellids distribution taken from Honěk (1982).

\begin{tabular}{ccccccccc}
\hline Model & Estimate & SE & TStat & PValue & $R^{2}$ & $\begin{array}{c}\text { Mean sum } \\
\text { of squares }\end{array}$ & FRatio & PValue \\
\hline$c \sqrt{K}$ & $c=2.1$ & 0.17 & 12.3 & $1.8 \times 10^{-14}$ & 0.8 & 1800 & 151 & $1.8 \times 10^{-14}$ \\
$c K^{2 / 3}$ & $c=0.45$ & 0.051 & 8.9 & $1.1 \times 10^{-10}$ & 0.69 & 2903 & 80 & $1.1 \times 10^{-10}$ \\
$c K$ & $c=0.017$ & 0.003 & 5.8 & $1.2 \times 10^{-6}$ & 0.5 & 4845 & 33 & $1.3 \times 10^{-6}$ \\
$c K^{2}$ & $c=6.7 \times 10^{-7}$ & $2.1 \times 10^{-7}$ & 3.2 & 0.003 & 0.23 & 7266 & 10.5 & 0.003 \\
\hline
\end{tabular}

\subsection{Immigration is proportional to patch quality}

Here I assume that immigration is directly proportional to patch quality $K_{i}$. This means that individuals can sense prey abundance, but their decision where to immigrate is not influenced by the number of conspecifics already present in the patch. The immigration rates are $a_{i}=\frac{K_{i}}{K_{1}+\ldots+K_{H}}$. When substituted to (3) the equilibrium distribution for emigration rates (6) is given by (9). Once again we get the Parker's matching principle.

For alternative emigration rates (7) the corresponding equilibrium is

$$
m_{i}=c K_{i}^{2} \text { for every } i=1, \ldots, H .
$$

Thus $m_{i} / m_{j}=K_{i}^{2} / K_{j}^{2}$ which corresponds to the "overmatching" because good patches contain a higher proportion of lady beetles than would correspond to the IFD.

\subsection{Immigration is proportional to patch payoff}

Another possibility is to consider the case where immigration rate is proportional to patch payoff $V_{i}$. In this case the dispersing individuals must be able not only to sense the number of aphids in a patch but also the number of conspecifics in that patch. The corresponding immigration rates are

$$
a_{i}=\frac{K_{i} / m_{i}}{K_{1} / m_{1}+\ldots+K_{H} / m_{H}} .
$$

When substituted to (3) the equilibrium distribution for emigration rates given by (6) is

$$
m_{i}=c K_{i}^{\frac{2}{3}} \text { for } i=1, \ldots, H
$$

Thus, $m_{i} / m_{j}=\left(K_{i} / K_{j}\right)^{\frac{2}{3}}$ which corresponds to undermatching.

For alternative emigration rates (7) the predicted distribution is given by the Parker's matching principle (9).

\section{WHICH MODEL FITS DATA BEST?}

Honěk (1982) (reprinted in Hodek \& Honěk, 1996) published data that relate density of Coccinella septempunctata to aphid density (Fig. 1). For these data I estimated the unknown parameter $c$ in models (8), (9), (10), (11) using regression function Regress of Mathematica 6 (Wolfram, 2003). Function Regress finds a least-squares fit to a list of data for a given linear combination of functions. For example, to estimate parameter $c$ for (8) I used command Regress[data, $\{\sqrt{x}\}, x$, IncludeConstant- $>$ False]. Without setting the optional argument IncludeConstant to False value, Mathematica would automatically estimate parameters $k$ and $c$ for model $k+c \sqrt{x}$. With this optional argument, the absolute term $k$ is not included. The results are given in Table 1 and Fig. 1. Because Fig. 1 plots the data in the log-log scale, line slopes correspond to exponents of corresponding models. As least-squares minimize the sum of squared errors the predicted values must fit observed data for high lady beetle densities quite well. Thus, most of the data are above the estimated lines in Fig. 1.
First, I will consider results for emigration rates given by (6). The corresponding distributions are then given by models (8), (9) and (11). Among these models, model (8) that corresponds to unconditional immigration (solid line in Fig. 1) fits the data best (in the sense of mean sum of squares, Table 1) while model (9) that corresponds to the IFD provides the worst fit (dotted line in Fig. 1).

Second, when emigration rates are given by (7), the corresponding distributions are given by models (9) and (10) because the model with unconditional immigration and the model with immigration proportional to patch payoff lead to the same distribution (9). Model (10) gives even worse fit (long-dashed line) than model (9).

In addition, I also estimated parameters for a general power function $m_{i}=c K_{i}^{d}$. Not surprisingly, the general power law with two estimated parameters $c=4.3$ and $d=$ 0.435 gives the best least-squares fit (dot-dashed line in Fig. 1, mean sum of squares $=1580)$.

\section{DISCUSSION}

In this article I studied animal dispersal using some hypotheses on dispersal rates that correspond to empirical observations on coccinellids. I assumed that emigration rates increase with decreasing patch payoffs defined either as the ratio between patch quality (measured by the number of aphids) and number of consumers, or as the patch quality only. It was observed in several studies on coccinellids that emigration rates increase with decreasing patch quality (Ives et al., 1993; Hodek \& Honěk, 1996;

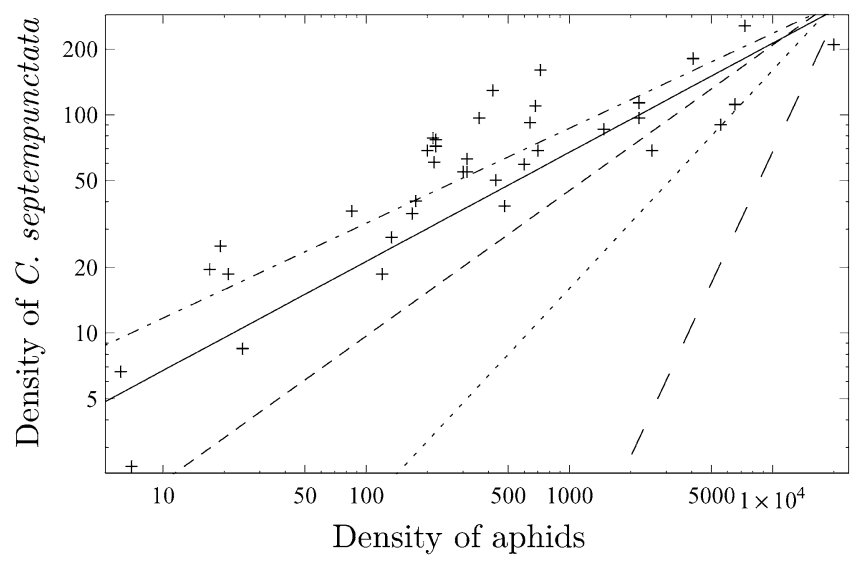

Fig. 1. This figure shows the relation between abundance of C. septempunctata adults and aphids (Acyrthosiphon pisum). Data (crosses) were taken from Honěk (1982). The solid line is for model (8), the short-dashed line for model (11), the dotted line for model (9), the long-dashed line for model (10) and the dash-dotted line for a general power law function $c K^{d}$ (with $c=$ 4.3 and $d=0.435$ ). Estimated values of $c$ are given in Table 1 . 
Osawa, 2000; Cardinale et al., 2006). It was also observed that searching efficiency decreases with increasing number of conspecifics (Siddiqui et al., 1999).

For immigration rates, I considered three scenarios: individuals chose patches unconditionally, immigration is proportional to patch quality, and immigration is proportional to patch payoff. These assumptions allowed me to make predictions on consumer distribution. All resulting distributions are given by a power law. Using data on distribution of Coccinella septempunctata with respect to various aphid densities taken from Honěk (1982) (see also Hodek \& Honěk, 1996) I tested which of these models fits the data best. Among six different patterns of dispersal studied in this article, the model where emigration is directly proportional to number of conspecifics and inversely proportional to number of aphids combined with unconditional immigration fit data from Honěk (1982) best (solid line in Fig. 1). This model corresponds to the so called "undermatching" where poor (good) patches contain higher (lower) proportion of C. septempunctata than would correspond to the IFD. This phenomenon was observed in several studies on animal distribution (e.g., Milinski, 1988; Kennedy \& Gray, 1993; Houston et al., 1995; Jackson et al., 2004). Honěk's data on lady beetles is another example of such a distribution. My predictions on the most plausible immigration and emigration rates agree well with Cardinale et al. (2006) who did not observe any correlation between immigration rates of three lady beetle species and prey density but observed a negative correlation between emigration rates and aphid density.

Fryxell et al. (2004) studied patterns of dispersal for Thomson's gazelles (Gazella thomsoni thomsoni Günter) on the Serengeti Plains of East Africa. They assumed that probability of emigration depends on the local energy gain relative to the expected rate of daily energy gain averaged over the landscape. Such a rule implicitly assumes that animals have a global knowledge of their environment. They investigated several modes of immigration rates and they found that gazelle movements were best predicted by the model where probability of immigration into neighboring sites was determined by relative rates of energy intake. The discrepancy between their predictions and predictions for lady beetles can be caused by several factors. First, both models differ in how emigration rates depend on patch quality. While my models assume that emigration depends only on the quality of the current patch, Fryxell et al. (2004) assume emigration rates to be dependent on both the quality of the local patch as well as the average quality of all patches. Second, the discrepancies can be due to different rules for herbivore vs. predator dispersal.

An important assumption made in this article is that the observed distribution corresponds to a stable equilibrium pattern of lady beetle dispersion among aphid patches. This requires that patch quality given by aphid numbers is at least temporarily fixed. Honěk's data used in this article come from a largely uniform habitat in which aphid and lady beetle populations had been building for a few weeks so that a temporary equilibrium in beetle distribution may well have been reached. Aphid local populations are often ephemeral (boom and bust) so on a longer time scale the assumption on fixed patch quality is not justified and aphid population dynamics must be taken into account.

ACKNOWLEDGMENTS. I wish to thank A. Honěk for sending me the original data on lady beetles distribution, I. Hodek who greatly helped me in thinking on this topic, E.W. Evans and an unknown reviewer for their suggestions that greatly improved this article. This work was supported by GA AV (IAA100070601). Institute of Entomology is funded by AS CR (Z50070508).

\section{REFERENCES}

BANKS C.J. 1957: The behavior of individual coccinellid larvae on plants. Anim. Behav. 5: 12-24.

Cardinale B.J., Weis J.J., Forbes A.E., Tilmon K.J. \& Ives A.R. 2006: Biodiversity as both a cause and consequence of resource availability: a study of reciprocal causality in a predator-prey system. J. Anim. Ecol. 75: 497-505.

Cressman R. \& KřIVAn V. 2006: Migration dynamics for the ideal free distribution. Am. Nat. 168: 384-397.

Doncaster C.P., Clobert J., Doligez B., Gustafsson L. \& DanCHIN E. 1997: Balanced dispersal between spatially varying local populations: An alternative to the source-sink model. Am. Nat. 150: 425-445.

Elliott N.C. \& Kieckhefer R.W. 2000: Response by coccinellids to spatial variation in cereal aphid density. Popul. Ecol. 42: 81-90.

Evans E.W. 2003: Searching and reproductive behaviour of female aphidophagous ladybirds (Coleoptera: Coccinellidae): a review. Eur. J. Entomol. 100: 1-10.

Evans E.W. \& Toler T.R. 2007: Aggregation of polyphagous predators in response to multiple prey: ladybirds (Coleoptera: Coccinellidae) foraging in alfalfa. Popul. Ecol. 49: 29-36.

Frazer B.D. 1988: Coccinellidae. In Minks A.K. \& Harrewijn P. (eds): Aphids, Their Biology, Natural Enemies and Control. Vol. B. Elsevier, Amsterdam, pp. 217-230.

Fretwell D.S. \& LuCAS H.L. 1970: On territorial behavior and other factors influencing habitat distribution in birds. Acta Biotheor. 19: 16-32.

FryXell J.M., Wilmshurst J.F. \& Sinclair A.R.E. 2004: Predictive models of movement by serengeti grazers. Ecology 85: 2429-2435.

Hastings A. 1983: Can spatial variation alone lead to selection for dispersal? Theor. Popul. Biol. 24: 244-251.

Hodek I. \& HonĚK A. 1996: Ecology of Coccinellidae. Kluwer Academic, Dordrecht, $464 \mathrm{pp}$

Holt R.D. 1985: Population dynamics in two-patch environments: Some anomalous consequences of an optimal habitat distribution. Theor. Popul. Biol. 28: 181-208.

Holt R.D. \& BARfield M. 2001: On the relationship between the ideal-free distribution and the evolution of dispersal. In Danchin J.C.E., Dhondt A. \& Nichols J. (eds): Dispersal. Oxford University Press, Oxford, pp. 83-95.

HoNĚK A. 1980: Population density of aphids at the time of settling and ovariole maturation in Coccinella septempunctata (Col.: Coccinellidae). Entomophaga 25: 427-430.

HoNĚK A. 1982: Factors which determine the composition of field communities of adult aphidophagous Coccinellidae (Coleoptera). Z. Angew. Entomol. 94: 157-168.

Houston A.I., McNamara J.M. \& Milinski M. 1995: The distribution of animals between resources - a compromise between 
equal numbers and equal intake rates. Anim. Behav. $248-251$.

Ives A.R., Kareiva P. \& Perry R. 1993: Response of a predator to variation in prey density at three hierarchical scales: lady beetles feeding on aphids. Ecology 74: 1929-1938.

Ives P.M. 1981: Estimation of Coccinellid numbers and movement in the field. Can. Entomol. 113: 981-997.

Jackson A.L., Ranta E., Lundberg P., Kaitala V. \& Ruxton G.D. 2004: Consumer-resource matching in a food chain when both predators and prey are free to move. Oikos 106: 445-450.

KenNedy M. \& GRAY R.D. 1993: Can ecological theory predict the distribution of foraging animals? A critical analysis of experiments on the ideal free distribution. Oikos 68: 158-166.

McPeek M.A. \& Holt R.D. 1992: The evolution of dispersal in spatially and temporally varying environments. Am. Nat. 140: 1010-1027.

MILINSKI M. 1988: Games fish play: making decisions as a social forager. Trends Ecol. Evol. 3: 325-330.

MilinsKi M. \& PARKER G.A. 1991: Competition for resources. In Krebs J.R. \& Davies N.B. (eds): Behavioural Ecology: An Evolutionary Approach. Blackwell, Oxford, pp. 137-168.

Osawa N. 2000: Population field studies on the aphidophagous ladybird beetle Harmonia axyridis (Coleoptera: Coccinellidae): resource tracking and population characteristics. Popul. Ecol. 42: 115-127.
Parker G.A. 1978: Searching for mates. In Krebs J.R. \& Davies N.B. (eds): Behavioural Ecology: An Evolutionary Approach. Blackwell, Oxford, pp. 214-244.

Parker G.A. \& Stuart R.A. 1976: Animal behavior as a strategy optimizer: Evolution of resource assessment strategies and optimal emigration thresholds. Am. Nat. 110: 1055-1076.

RŮŽIČKA Z. \& ZEMEK R. 2007: Deterrent effects of larval tracks on conspecific larvae in Cycloneda limbifer. BioControl 10.1007/s10526-007-9109-x.

Siddiqui A., Kumar A., Kumar N. \& Tripathi C. 1999: Preypredator relationship between Lipaphis erysimi Kalt. (Homoptera: Aphididae) and Coccinella septempunctata Linn. (Coleoptera: Coccinellidae): III. Effect of host plants on the searching strategy, mutual interference and killing power of the predator. Biol. Agric. Hortic. 17: 11-17.

Sutherland W.J. 1996: From Individual Behaviour to Population Ecology. Oxford University Press, Oxford, 224 pp.

Turchin P. \& Kareiva P. 1989: Aggregation in Aphis varians: an effective strategy for reducing predation risk. Ecology 70: 1008-1016.

van der Werf W., Evans E.W. \& Powell J. 2000: Measuring and modelling the dispersal of Coccinella septempunctata (Coleoptera: Coccinellidae) in alfalfa fields. Eur. J. Entomol. 97: 487-493.

Wolfram S. 2003: The Mathematica Book. Wolfram Media/ Cambridge University Press, 1488 pp.

Received September 18, 2007; revised and accepted November 26, 2007 